\title{
Drop formation in microfluidic cross-junction: jetting to dripping to jetting transition
}

\author{
Nina M. Kovalchuk ${ }^{1}\left[\right.$ [ $\cdot$ Masanobu Sagisaka ${ }^{2} \cdot$ Kasparas Steponavicius $^{1} \cdot$ Daniele Vigolo $^{1} \cdot$ Mark J. H. Simmons $^{1}$
}

Received: 29 January 2019 / Accepted: 23 July 2019 / Published online: 31 July 2019

(c) The Author(s) 2019

\begin{abstract}
The regimes of drop generation were studied in a Dolomite microfluidic device which combined both hydrodynamic and geometrical flow focusing over a broad range of flow rates. A series of aqueous dispersed phases were used with a viscosity ratio between continuous and dispersed phases of close to unity. Surfactants were added to alter the interfacial tension. It was shown that the transition from dripping to jetting is well described by the capillary numbers of both the dispersed and continuous phases. Only the jetting regime was observed if the capillary number of the dispersed phase was above a critical value, whereas at smaller values of this parameter a jetting $\rightarrow$ dripping $\rightarrow$ jetting transition was observed by increasing the capillary number of the continuous phase. The analysis performed has shown that the conditions for a dripping to jetting transition at moderate and large values of the capillary number of the continuous phase can be predicted theoretically by comparison of the characteristic time scales for drop pinch-off and jet growth, whereas the transition at small values cannot. It is suggested that this transition is geometry mediated and is a result of the interplay of jet confinement in the focusing part and a decrease of confinement following entry into the main channel. The flow fields inside the jet of the dispersed phase were qualitatively different for small and large values of the capillary number of the continuous phase revealing the relative contribution of the dispersed phase flow in jet formation. The volume of the drops formed in the jetting regime increased as a power law function of the flow rate ratio of the dispersed to continuous phase, independent of the interfacial tension.
\end{abstract}

Keywords Flow focusing $\cdot$ Flow map $\cdot$ Regime transitions $\cdot$ Drop size $\cdot$ Interfacial tension $\cdot$ Capillary number

\section{Introduction}

Drop microfluidics is a rapidly developing area enabling considerable scale reduction for synthetic and analytical processes by manipulation of drops with volumes in the nanoand picolitre range (DeMello 2006; Whitesides 2006; Marre and Jensen 2010; Rakszewska et al. 2014; Holtze et al. 2017; Vladisavljević et al. 2017). Drops can be formed using coflow of dispersed and continuous phases (Herrada et al.

Electronic supplementary material The online version of this article (https://doi.org/10.1007/s10404-019-2269-z) contains supplementary material, which is available to authorized users.

Nina M. Kovalchuk

n.kovalchuk@bham.ac.uk

1 School of Chemical Engineering, University of Birmingham, Birmingham B152TT, UK

2 Graduate School of Science and Technology, Hirosaki University, Hirosaki 036-8561, Japan
2008; Utada et al. 2008; Capretto et al. 2012; Mitropoulos et al. 2014), cross-flow in T-junction (Garstecki et al. 2006; Christopher et al. 2008; van Steijn et al. 2010; Glawdel et al. 2012) and flow focusing. Flow focusing has become the most popular method of drop production in flow microfluidics because it allows the formation of highly monodisperse drops over a wide size range using devices which are easily designed by soft lithography. According to the Web of Science, the number of articles published on flow-focusing microfluidics over the last 2 years was four and ten times larger than for T-junction and co-flow designs, respectively. Flow focusing can be achieved in various ways: by interaction of the flows of dispersed and continuous phases in a cross-junction (hydrodynamic focusing) (Cubaud and Mason 2008; Carrier et al. 2015; Chen et al. 2015), by passing coflowing dispersed and continuous phases through an orifice (geometrical focusing) (Anna et al. 2003; Garstecki et al. 2005; Lee et al. 2009), by combination of hydrodynamic and geometrical focusing (Ward et al. 2010; Kovalchuk et al. 
2018b) or by optical focusing (de Saint Vincent and Delville 2016).

Flow-focusing devices produce drops in four different regimes which are denoted as squeezing, dripping, jetting and tip streaming (Anna and Mayer 2006; Cubaud and Mason 2008; Lee et al. 2009; Kovalchuk et al. 2018b). The squeezing regime usually results in the formation of plugs with a length larger than the channel width whilst smaller drops are formed in the dripping regime. The jetting regime covers a range of drop sizes which are similar to both dripping and squeezing, whereas tip streaming enables the formation of a train of very small droplets, much smaller than the channel size, periodically following the formation of a main drop of the order of the channel size.

The main requirement for drops serving as isolated chemical reactors, precursors for drug-loaded capsules or a controlled environment for biological objects, is monodispersity. Reliable size control is thus required, often when the fluid properties of the dispersed phase, such as viscosity and interfacial tension, are fixed. It is generally accepted that drops with uniform size distribution can be produced in a microfluidic device operating in the squeezing and dripping regime, which is related to the absolute instability. Conversely, in the jetting regime, where drops are formed due to convective instability, a broader size distribution is observed (Cordero et al. 2011; Chen et al. 2013). Therefore, finding the parameter space where drops are formed in the squeezing/dripping regime (thereafter referred to as dripping regime) is of critical importance and many microfluidic studies have been carried out to produce such flow maps (Anna and Mayer 2006; Cubaud and Mason 2008; Fu et al. 2012; Shahriari et al. 2016; Mastiani et al. 2017; Wu et al. 2017; Kovalchuk et al. 2018b).

In the simplest case of liquid flow in an unconfined inviscid environment, transition from dripping to jetting is determined by the competition of liquid inertia and surface tension described by the Weber number $W e=\frac{\rho V^{2} L}{\sigma}$, where $\rho$ is the liquid density, $V$ is the liquid velocity, $L$ is the characteristic length scale (normally the radius of the nozzle from which the liquid is flowing) and $\sigma$ is the surface tension. However, even in this case the critical value of the Weber number $W e_{\text {cr }}$ depends on the liquid viscosity, the relative importance of gravity represented by the Bond number and the thickness of the nozzle wall (Clanet and Lasheras 1999; Ambravaneswaran et al. 2004). In particular, an increase in viscosity results in a decrease of $W e_{\mathrm{cr}}$ (Ambravaneswaran et al. 2004).

Adding a viscous outer (continuous) liquid phase complicates things further. The moving continuous phase applies a drag force to the dispersed phase causing a transition to jetting at a certain threshold value of the continuous phase velocity (Cramer et al. 2004; Utada et al. 2007). Therefore, an additional parameter, the capillary number of continuous phase, $C a_{\mathrm{c}}=\frac{\mu_{\mathrm{c}} V_{\mathrm{c}}}{\sigma}$, comes into play. Here, $\mu_{\mathrm{c}}$ is the dynamic viscosity of continuous phase and $\mathrm{V}_{\mathrm{c}}$ is the velocity of continuous phase. In Cramer et al. (2004), the transition from dripping to jetting for unconfined co-flow was observed only if the velocity of the continuous phase exceeded a certain threshold value. A more sophisticated flow map was found for the co-flow of liquid phases over a broad range of viscosity ratios (0.01-10) in Utada et al. (2007), where also a large ratio between the diameter of the channel for the continuous phase and the nozzle for the dispersed phase was employed. According to Utada et al. (2007), the dripping regime is observed only under the condition of $W e_{\mathrm{d}}<W e_{\mathrm{cr}} \cap$ $C a_{\mathrm{c}}<C a_{\mathrm{cr}}$ If the Weber number of the dispersed phase, $W e_{\mathrm{d}}$, exceeded the threshold value, $W e_{c r}$, the jetting regime was observed over the whole studied range of capillary number of the continuous phase. In this case, the transition to jetting is driven by the inertia of the dispersed phase. The regime is characterised by a widening jet and a drop size larger than the nozzle size. On the other hand, if the capillary number of the continuous phase, $C a_{\mathrm{c}}$ exceeded the threshold value, and $C a_{\text {cr }}$, a jetting regime driven by the viscous drag imposed by the continuous phase, was observed independently of $W e_{\mathrm{d}}$. In this case, the jet narrows down the channel and the drop size is smaller than the nozzle. Similar results were obtained also in Chen et al. (2013) where the experimental data obtained were compared with numerical simulations.

For the case when the drop formation under co-flow occurs under confinement, i.e. the drop size is comparable to the size of continuous phase channel, the dripping area of the flow map is found to be no longer rectangular. According to the theoretical analysis confirmed by the experimental study in Guillot et al. (2007), the transition line between dripping and jetting becomes curvilinear and for flow rates of dispersed phase smaller than a threshold value, the transition jetting $\rightarrow$ dripping $\rightarrow$ jetting was found by the increase in the flow rate of continuous phase. In flow-focusing devices, the sizes of input channels for dispersed and continuous phase as well as the size of the output channel are of the same order of magnitude and the drop size is comparable to the channel size. In this case, not only does the continuous phase affect the flow field inside the dispersed phase, but the forming drop of the dispersed phase affects considerably the flow field of continuous phase, by decreasing the available cross-section of the output channel through which it can flow. Thus, it can be expected that the flow maps reflecting the transition from dripping to jetting in flow-focusing devices are more complicated than those reported in Utada et al. (2007) and can be similar to that reported in Guillot et al. (2007). This is surprising, as to our knowledge, that the transition jetting $\rightarrow$ dripping $\rightarrow$ jetting was not previously observed in flow-focusing devices.

In a number of studies (Cubaud and Mason 2008; Fu et al. 2012; Shahriari et al. 2016; Mastiani et al. 2017; Kovalchuk 
et al. 2018b), the transition from dripping to jetting in various microfluidic flow-focusing devices was observed by an increase in the flow rate of the dispersed phase but in $\mathrm{Wu}$ et al. (2017), this transition was reported to happen by an increase in the flow rate of both continuous and dispersed phases, whereas in Anna and Mayer (2006) it was observed only by an increase of the flow rate of the continuous phase. Besides in most studies (Fu et al. 2012; Shahriari et al. 2016; Mastiani et al. 2017; Wu et al. 2017; Kovalchuk et al. $2018 \mathrm{~b}$ ), the critical flow rate of the transition-defining phase was dependent on the flow rate of the second phase, whereas in Anna and Mayer (2006), Cubaud and Mason (2008) it was practically constant.

The discrepancies between the various studies on the regime transition from dripping to jetting in microfluidic flow-focusing devices demonstrate the lack of understanding of the precise mechanisms and the necessity for further thorough study. Here, we present the results of such a study for the case when the viscosity ratio of the dispersed to the continuous phase is of order of unity, the condition being similar to Wu et al. (2017), Kovalchuk et al. (2018b). To prove the generality of the obtained results, the interfacial tension between the dispersed and continuous phases was systematically varied over a large range by addition of surfactants. Further attention was paid to the size of the drops formed in the jetting regime.

\section{Materials and methods}

The cationic surfactant dodecyltrimethylammonium bromide, $\mathrm{C}_{12} \mathrm{TAB}$, critical micelle concentration, $\mathrm{cmc}=16 \mathrm{mM}$ (Across organics, 99\%) and non-ionic surfactant Triton $\mathrm{X}-100, \mathrm{TX}-100, \mathrm{cmc}=0.6 \mathrm{mM}$ (Sigma-Aldrich, laboratory grade); glycerol (Alfa Aesar, ultrapure, HPLC grade); and silicone oil, viscosity standard $5 \mathrm{cSt}$ (Aldrich) were used without additional purification. Anionic surfactant sodium bis(2,6-dimethyl-4-heptyl)-2-sulfoglutarate, di-BC $\mathrm{C}_{9} \mathrm{SG}$, $\mathrm{cmc}=4.3 \mathrm{mM}$ was synthesised and purified as described in Sagisaka et al. (2014). The purity of the di-BC ${ }_{9} \mathrm{SG}>99 \%$ was confirmed by HPLC and elemental analysis. Doubledistilled water was produced by a still (Aquatron A 4000 D, Stuart).

A surfactant was dissolved in the aqueous (dispersed) phase, being a mixture of $52 \%$ glycerol and $48 \%$ water (G_W) by mass. The same concentration, $50 \mathrm{mM}$, was used for all surfactants in the flow map studies. This concentration is far above the cmc for all surfactants under study. Additionally, several concentrations of di-BC ${ }_{9} \mathrm{SG}$ below and at the cmc were used to study the drop size. Silicone oil was used as the continuous phase. The continuous and dispersed phase had matched refractive indices, $n=1.403$, which enabled avoidance of optical distortions near the interface while measuring the flow fields inside the dispersed phase. The ionic surfactants used are insoluble in the continuous phase. The non-ionic surfactant is soluble; however, its partition is considerably in favour of the aqueous phase and the mass transfer to the oil phase can be neglected on the sub-second timescale of the experiments in this study. The physical properties of the continuous and dispersed phases used are given in Table 1. To enhance optical contrast, methyl violet dye was added to the aqueous phase at a concentration $\sim 1 \mathrm{~g} / \mathrm{L}$. The dye addition did not affect the interfacial tension between the oil and aqueous phases (Kovalchuk et al. 2018b).

Experiments were performed using a glass Droplet Junction Chip (cross-junction) with a hydrophobised inner surface (Dolomite Microfluidics, UK). The geometry, as presented in the Dolomite Product Datasheet, is shown in Fig. 1. The liquids were supplied to the chip using syringe pumps Al-4000 (World Precision Instruments, UK), equipped with $10 \mathrm{~mL}$ syringes (BD Plastipak ${ }^{\mathrm{TM}}$ ) for flow rates, $Q \geq 1 \mu \mathrm{L} / \mathrm{min}$ and with $1 \mathrm{~mL}$ syringes (BD Plastipak $^{\mathrm{TM}}$ ) for $Q \leq 6 \mu \mathrm{L} / \mathrm{min}$. The same types of syringes were used for both continuous and dispersed phases and consistent results were obtained with both types of syringes in the range $1 \mu \mathrm{L} / \mathrm{min} \leq Q_{\mathrm{c}, \mathrm{d}} \leq 6 \mu \mathrm{L} / \mathrm{min}$. Here, $Q_{\mathrm{d}}$ is defined as the flow rate of dispersed phase supplied through the central input channel and $Q_{\mathrm{c}}$ is the flow rate of the continuous phase in each of the side input channels, i.e. the total
Table 1 Physical properties of the liquid phases

\begin{tabular}{llll}
\hline Liquid & Density $\left(\mathrm{kg} / \mathrm{m}^{3}\right)$ & $\begin{array}{l}\text { Dynamic viscosity } \\
(\mathrm{mPa} \mathrm{s})\end{array}$ & $\begin{array}{l}\text { Equilibrium } \\
\text { interfacial tension } \\
(\mathrm{mN} / \mathrm{m})\end{array}$ \\
\hline Silicone oil, continuous phase & 920 & 4.6 & 29 \\
Surfactant-free G_W, disperse phase & 1133 & 6.4 & 29 \\
50 mM of C ${ }_{12}$ TAB in G_W & 1133 & 6.4 & 10 \\
50 mM TX-100 in G_W & 1133 & 6.4 & 3.6 \\
2.15 mM di-BC 9 SG in G_W & 1133 & 6.4 & 6.7 \\
4.3 mM di-BC 9 SG in G_W & 1133 & 6.4 & 4.2 \\
50 mM di-BC 9 SG in G_W & 1133 & 6.4 & 1.4 \\
\hline
\end{tabular}


Fig. 1 Microfluidic chip geometry. Adapted from the Dolomite Product Datasheet with permission from Dolomite Microfluidics, UK

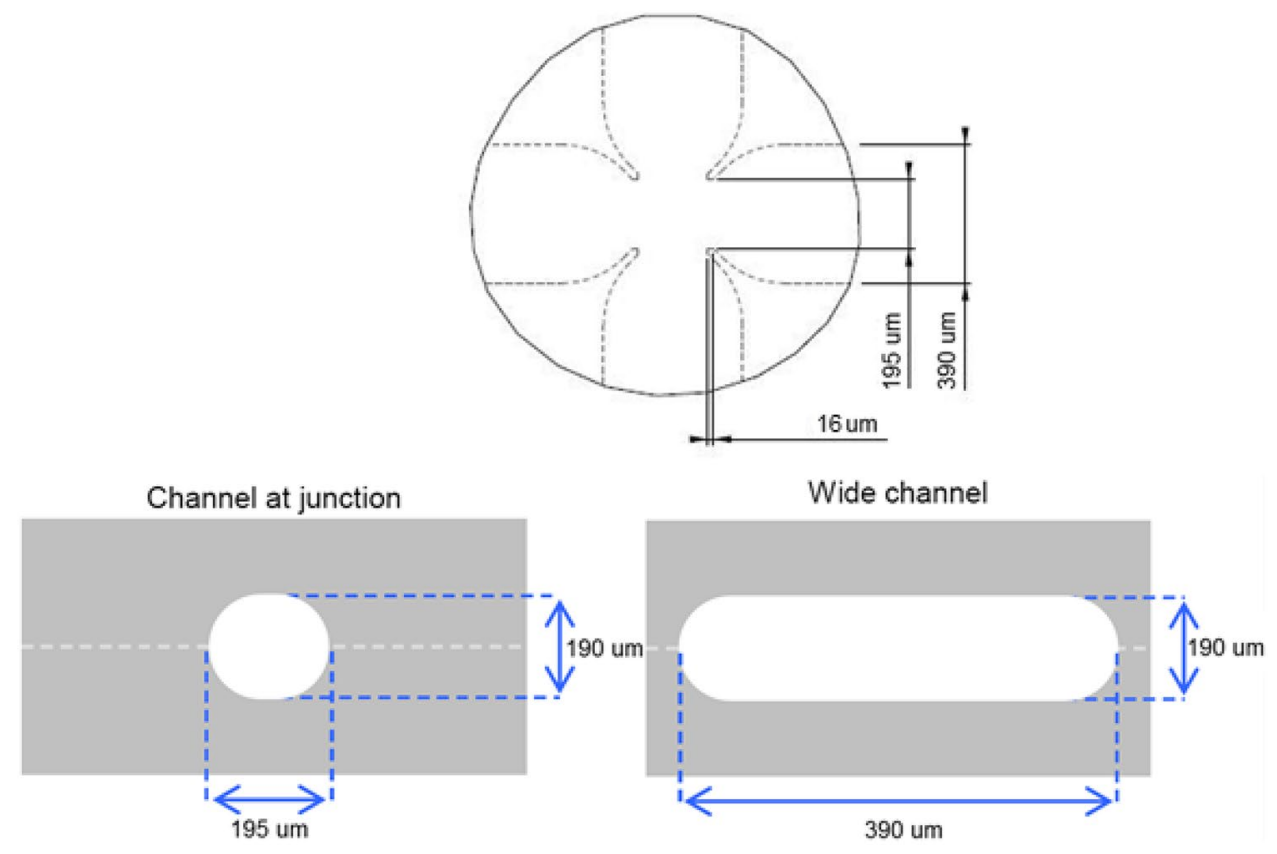

flow rate in the output channel $Q_{\mathrm{s}}=Q_{\mathrm{d}}+2 Q_{\mathrm{c}}$. After any change in flow rate, the system was allowed to stabilize for $0.5-10 \mathrm{~min}$ depending on flow rate.

As the transition between the dripping and jetting regimes of drop formation exhibits noticeable hysteresis (Clanet and Lasheras 1999; Cramer et al. 2004), in this study the threshold values of flow rates were determined by moving from dripping to jetting. To find the transition, the flow rate of continuous phase was kept constant whilst the flow rate of dispersed phase was increased from its minimum value. The increment depended on the flow rate and the presence of surfactant: it was smaller for the surfactant solutions providing smaller interfacial tension. For the surfactant-free system, the increment was normally $2-4 \mu \mathrm{L} / \mathrm{min}$ at flow rates up to $10 \mu \mathrm{L} / \mathrm{min}$ and $10 \mu \mathrm{L} / \mathrm{min}$ thereafter. For selected $Q_{\mathrm{d}}$, experiments were repeated by increasing the flow rate of the continuous phase starting from the $Q_{\mathrm{c}}$ in the region of jetting regime dominated by the dispersed phase. The obtained transition points were in good agreement with those obtained in experiments by varying the flow rate of the dispersed phase.

The complex chip geometry, with varying cross-sectional area, allows several definitions of velocity for the capillary number. In the following, the capillary numbers for dispersed and continuous phase were calculated using superficial velocities in the feeding channels, $U_{\mathrm{c}}=Q_{\mathrm{c}} / S$ and $U_{\mathrm{d}}=Q_{\mathrm{d}} / S$, where $S=6.63 \times 10^{-8} \mathrm{~m}^{2}$ is the area of the channel cross-section. Capillary numbers based on the velocities in the flow-focusing parts are $\sim 2.3$ times larger and $C a_{\mathrm{c}}$ based on the axial elongation rate (Anna and Mayer 2006; Anna 2016) is 1.3 times larger than the values used in this study.
The flow regimes were monitored at $2000 \mathrm{fps}$ using a high-speed video camera (Photron SA5) equipped with a Navitar, 2X F-mount objective giving a spatial resolution of $4 \mu \mathrm{m} / \mathrm{pixel}$. The kinetics of drop formation was studied with a high-speed video camera connected to an inverted microscope (Nikon eclipse Ti-U) at 20,000 fps with an exposure time set to $0.02 \mathrm{~ms}$. A $20 \times$ objective (Nikon, CFI Plan Fluor DLL) was used giving an image resolution of $1 \mu \mathrm{m} /$ pixel. Image processing was performed using the ImageJ free software (Schneider et al. 2012). All results obtained are an average of between 3 and 5 experiments. For the transition from dripping to jetting, often both regimes were observed around the transition flow rate. The flow rate was ascribed to the jetting regime, if jetting was observed in at least two experiments at this flow rate.

The flow fields inside the dispersed phase were studied using Ghost Particle Velocimetry (GPV) (Buzzaccaro et al. 2013; Kovalchuk et al. 2018a; Riccomi et al. 2018). This technique uses the speckle patterns produced by light scattered by particles smaller than the diffraction limit as a flow tracer. In this study, $200 \mathrm{~nm}$ polystyrene particles were used (10\% solid, Sigma), diluted in the dispersed phase at a ratio of 1:50 (v:v). The small size of the tracers and their low concentration ensured the non-intrusive nature of the measurement. Additionally, GPV permitted an accurate evaluation of the flow fields close to the interface between the dispersed and continuous phase.

The particles used as tracers generate a pattern presenting a uniform distribution of speckles that reflects the distribution of the nanoparticles. This is advantageous when compared to standard PIV measurements where the fluctuations of particle density at the interface can affect the spatial 
resolution. Conversely, the cross-correlation of the speckle pattern gave accurate values for the flow velocities in any regime explored with a resolution of about $15 \times 15 \mu \mathrm{m}^{2}$ up to the boundary between the two phases. The video recording was carried out using a high-speed camera connected to the microscope at $10000 \mathrm{fps}$ with an exposure time of $0.05 \mathrm{~ms}$. At least 100 frames were recorded. Images were processed by ImageJ to remove background noise (Pirbodaghi et al. 2015) and then analysed using the open-source MATLAB toolbox PIVlab (Thielicke and Stamhuis 2014).

The equilibrium interfacial tension was measured using a tensiometer K100 (Krüss) equipped with a Du Noüy platinum ring and a pendant-drop tensiometer made in-house using a gauge 28 needle (o.d. $0.362 \mathrm{~mm}$ and i.d. $0.184 \mathrm{~mm}$ ) (Sagisaka et al. 2008).

\section{Results and discussion}

Figure 2a depicts the transition lines between the dripping and jetting regimes in the parametric space of capillary numbers of the continuous and dispersed phases, respectively, for surfactant-free and three surfactant-laden dispersed phases, all with the same surfactant concentration of $50 \mathrm{mM}$. The choice of capillary numbers as the dimensionless parameters used in this study will be discussed later. Note that the surfactant concentration used, $50 \mathrm{mM}$, is much higher than the $\mathrm{cmc}$ for all surfactant-laden solutions; therefore, the dynamic effects of the surfactant can be neglected, as shown in Kovalchuk et al. (2018b) for $\mathrm{C}_{12} \mathrm{TAB}$, the surfactant with the highest $\mathrm{cmc}$ value. Therefore, the equilibrium interfacial tension was used to calculate the capillary numbers. The experimental error for the transition points does not exceed $\Delta C a=5 \times 10^{-4}$. Although there is a certain shift of the transition lines to larger $C a_{\mathrm{c}}$ and smaller $C a_{\mathrm{d}}$ with a decrease of the interfacial tension, Fig. 2a shows that the transition from dripping to jetting can be described reasonably well using the capillary numbers of the dispersed and continuous phases as parameters. The difference between the curves is much larger if the Weber number of the dispersed phase is used as a parameter.

According to Fig. 2a, dripping is limited to small values of the capillary numbers of the dispersed phase and small to moderate capillary numbers of the continuous phase. The transition lines have a trapezoidal shape: the critical value of $C a_{\mathrm{d}}$ at transition, $C a_{\mathrm{dcrit}}$, increases with an increase of $C a_{\mathrm{c}}$ at small $C a_{\mathrm{c}}$ (ascending branch), is constant at moderate $C a_{\mathrm{c}}$ (plateau) and decreases with an increase in $C a_{\mathrm{c}}$ at larger $C a_{\mathrm{c}}$ (descending branch). If $C a_{\mathrm{d}}$ is below the critical value for the jetting region, $C a_{\text {dcrit } 0} \sim 6 \times 10^{-3}$, an increase of $C a_{\mathrm{c}}$ results in the transition jetting $\rightarrow$ dripping $\rightarrow$ jetting illustrated by Fig. 3. Note that the transition from dripping to jetting by an increase in the capillary number was not found in Kovalchuk
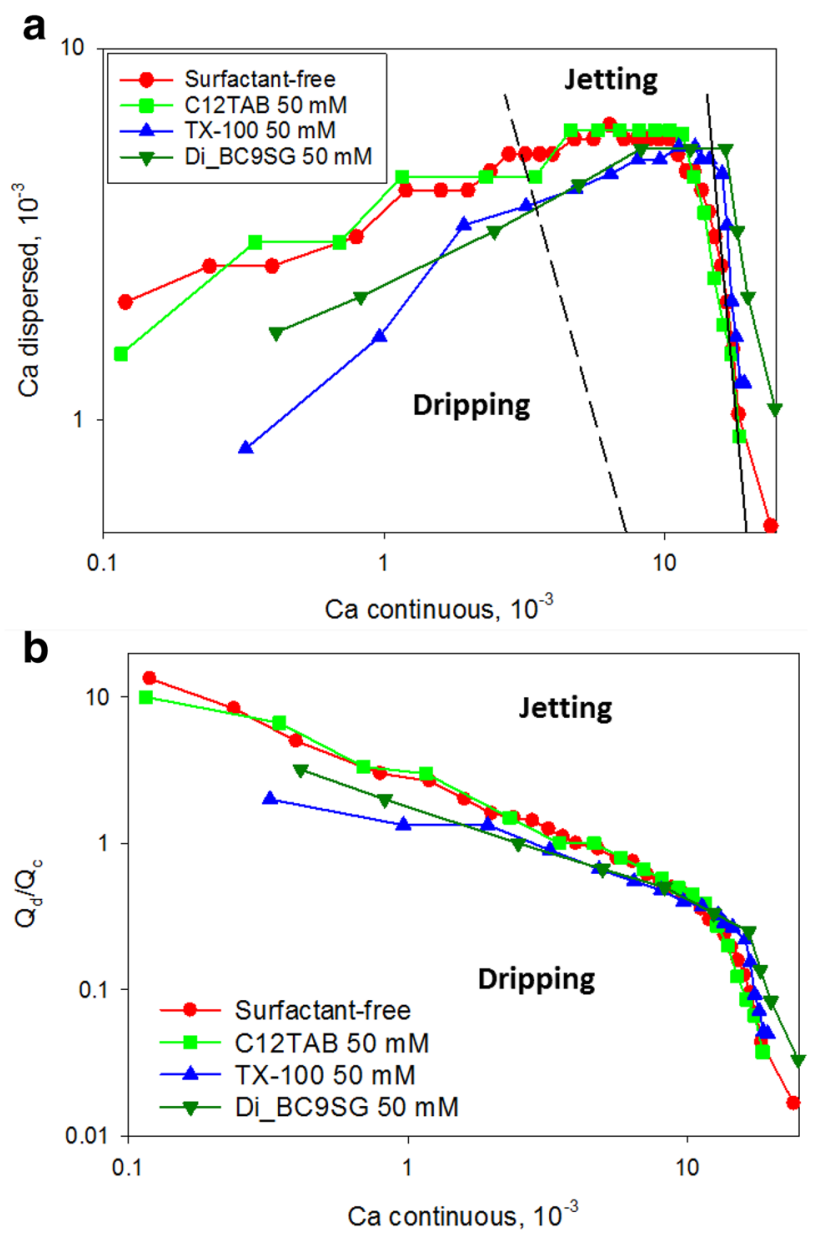

Fig. 2 Flow map depicting transition from dripping to jetting regime: the area below curves corresponds to dripping regime and the area at and outside curves correspond to jetting regime of drop formation. The solid line in Fig. 2a is a plot of inequality 7 (see below) at $C=25$, and the dashed line is a plot for $C=65$

et al. (2018b) despite a similar system being used, due to the limited range of capillary number of continuous phase examined in that study.

Redrawing Fig. 2a in coordinates $C a_{\mathrm{c}}-Q_{\mathrm{d}} / Q_{\mathrm{c}}$ (Fig. 2b) shows that the flow rate ratio below which the dripping regime was observed decreases with an increase of flow rate of the continuous phase $\left(C a_{\mathrm{c}}\right)$. Ascending and horizontal branches in Fig. 2a correspond to a slow monotonic decrease from $Q_{\mathrm{d}} / Q_{\mathrm{c}}=10$ at $C a_{\mathrm{c}}=10^{-4}$ to $Q_{\mathrm{d}} / Q_{\mathrm{c}} \sim 0.2-0.3$ at the end of the horizontal branch. Afterwards, the slope of the transition line increases considerably, indicating the change in the governing force responsible for the transition from dripping to jetting. It is noticeable that in the range of $C a_{\mathrm{c}}$ where the transition is governed by the flow of the dispersed phase (ascending branch and plateau in Fig. 2a), the drop size generated in the jetting regime at transition is larger than both the channel height and the diameter of the focusing channel. However, the drops formed at transition 


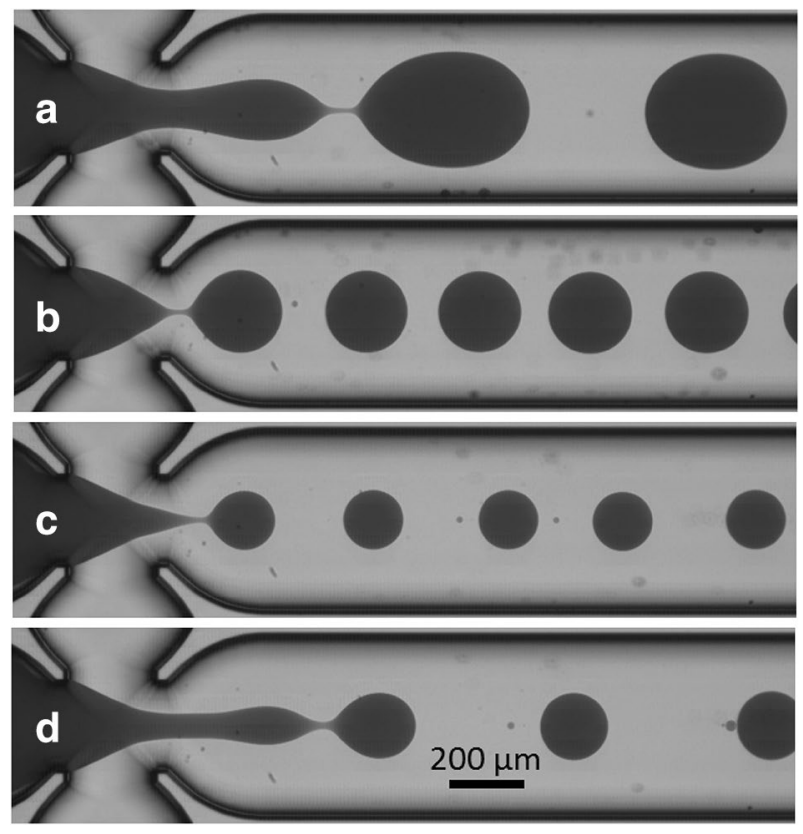

Fig. 3 Transition from jetting to dripping and again to jetting by an increase in the flow rate of the continuous phase. Dispersed phase is solution of $50 \mathrm{mM}$ TX-100 in $52 \%$ GL_W. Flow rate of the dispersed phase $Q_{\mathrm{d}}=9 \mu \mathrm{L} / \mathrm{min}\left(C a_{\mathrm{d}}=3.8 \times 10^{-3}\right)$, flow rates of the continuous phase: a $Q_{\mathrm{c}}=15 \mu \mathrm{L} / \mathrm{min}\left(C a_{\mathrm{c}}=4.8 \times 10^{-3}\right), \quad$ b $Q_{\mathrm{c}}=18 \mu \mathrm{L} /$ $\min \left(C a_{\mathrm{c}}=5.8 \times 10^{-3}\right), \quad$ c $Q_{\mathrm{c}}=45 \mu \mathrm{L} / \mathrm{min} \quad\left(C a_{\mathrm{c}}=14.4 \times 10^{-3}\right)$, d $Q_{\mathrm{c}}=50 \mu \mathrm{L} / \mathrm{min}\left(\mathrm{Ca}_{\mathrm{c}}=16.1 \times 10^{-3}\right)$

along the descending branch are smaller than both the channel height and the diameter of the focusing channel (Fig. 3).

Comparing Fig. 2a with flow maps available in the literature, it can be assumed that in Cramer et al. (2004) only the descending branch of transition was found for co-flow in a weakly confined geometry at $C a_{\mathrm{c}}>0.1$ and viscosity ratio $\mathrm{O}(1)$, in good agreement with our results. Two different mechanisms of transition from dripping to jetting were found in Utada et al. (2007): at $C a_{\mathrm{c}}<\mathrm{O}(1)$, the transition was driven by the inertia of the dispersed phase and occurred at $W e_{\mathrm{d}}=\mathrm{O}(1)$, whereas at $C a_{\mathrm{c}}>\mathrm{O}(1)$ the transition was driven by the viscous drag from the continuous phase. Thus, the dripping regime was possible only under the condition $W e_{\mathrm{d}}<\mathrm{O}(1) \cap C a_{\mathrm{c}}<\mathrm{O}(1)$. Our results are in line with (Utada et al. 2007); however, in the flow-focusing device, the range of parameters for the dripping regime is restricted to $C a_{\mathrm{d}}<\mathrm{O}\left(10^{-2}\right) \cap \mathrm{O}\left(10^{-3}\right)<C a_{\mathrm{c}}<\mathrm{O}\left(10^{-1}\right)$ and the critical capillary (Weber) number of the dispersed phase at transition depends on the capillary number of the continuous phase.

The sequence of regimes arising by an increase of the flow rate of continuous phase, keeping the flow rate of dispersed phase constant and below $C a_{\text {dcrito }}$, is shown in Fig. 3 . The corresponding videos are presented in the Electronic Supplementary Material (S1-S4). Note, in co-flow, the jet formed due to the inertia of the dispersed phase widens in the downstream direction as its (initially very high) average velocity adjusts to the equilibrium two-phase flow profile, whereas the (slower) jet formed due to viscous drag from the continuous phase narrows in the downstream direction. Figure 3 shows that in the flow-focusing device considered here the jet shapes are identical for both types of jetting: the jets become wider after leaving the focusing part of the channel to adapt to the wider channel.

An increase of the flow rate of the continuous phase at constant $Q_{\mathrm{d}}$, i.e. a decrease of $Q_{\mathrm{d}} / Q_{\mathrm{c}}$ results in a decrease of the drop size in both the dripping and jetting regimes. In the jetting regime, it is due to a decrease of the jet radius, which is proportional to $\left(Q_{\mathrm{d}} / Q_{\mathrm{c}}\right)^{1 / 2}$ (Cubaud and Mason 2008). In the dripping regime with the drop length larger than the channel width (often called squeezing regime), it is due to a faster growth of excessive pressure build-up in the continuous phase, due to the obstruction caused by the forming drop of the dispersed phase (Garstecki et al. 2005). When the drop length is noticeably smaller than the channel width (Fig. 3b, c), the shear stress from the continuous phase becomes the driving force for drop detachment (Umbanhowar et al. 2000) and the decrease in the drop size is due to an increase in the drag force per unit of area of the growing drop. Figure 3 shows, however, that the changes can be opposite at regime transition. In particular, the transition from dripping to jetting at high $C a_{\mathrm{c}}$ results in an increase in the drop size (sf. Fig. 3c, d). Such an increase was not observed in co-flow, where the transition to jetting occurred when the size of the drop formed in the dripping regime decreased to the nozzle size and the size of the drops formed in the jetting regime was always smaller than the nozzle (Utada et al. 2007). For the flow-focusing device, the increase can be attributed to the decrease in the superficial velocity of the continuous phase at the position of drop formation: in Fig. 3c, the drop is formed in a narrower part of the channel than in Fig. 3d. The change in the superficial velocity of the continuous phase can also explain an abrupt decrease in the drop size at the transition from jetting to dripping at small $\mathrm{Ca}_{\mathrm{c}}$. This results in an additional increase of superficial velocity of the continuous phase as the formed drop moves from the wide straight channel to the smaller transitional channel.

For flow-focusing devices based on the cross-junction geometry, the transition to drop formation in the jetting regime was found to occur at a nearly constant value of $C a_{\mathrm{d}} \sim 10^{-1}$ at $10^{-3}<C a_{\mathrm{c}}<3 \times 10^{-2}$ in Cubaud and Mason (2008). A slightly descending transition line with threshold value of $W e_{\mathrm{d}}$ decreasing from $10^{-1}$ to $10^{-2}$ when $C a_{\mathrm{c}}$ increases from $10^{-2}$ to $5 \times 10^{-2}$ was found in $\mathrm{Fu}$ et al. (2012). The ascending branch in these studies corresponds to the transition from dripping to tubing, the regime where a tube of the continuous phase occupies most of the channel cross-section and slightly invades the input channels of 
the continuous phase. The transition to the tubing regime in Cubaud and Mason (2008), Fu et al. (2012) instead of jetting observed in the present study can result from much higher viscosity ratio and the difference in geometry, as only hydrodynamic focusing in the cross-junction was used in Cubaud and Mason (2008), Fu et al. (2012). It can be assumed, however, that the difference in geometry is the most important parameter because in Shahriari et al. (2016) the ascending branch with transition to jetting was reported for the case of high viscosity ratio in a flowfocusing device of more sophisticated geometry including geometrical flow-focusing, whereas transition to tubing/ threading along the ascending branch was observed in Wu et al. (2017) for a viscosity ratio of 0.6. Transition from dripping to jetting was found in $\mathrm{Wu}$ et al. (2017) at $C a_{\mathrm{c}}>2 \times 10^{-3}$ demonstrating a plateau and partially descending branch in good agreement with our study. In a flow-focusing device with only geometrical focusing, the transition to jetting for a surfactant-free dispersed phase was observed only at $C a_{\mathrm{c}}>0.2$, independent of flow rate ratio (Anna and Mayer 2006). Transition to the jetting caused by the inertia of dispersed phase was not found in (Anna and Mayer 2006) due to the low values of We studied.

According to Utada et al. (2005), conditions for transition from dripping to jetting can be estimated by comparison of the time scale of drop pinch-off with the time scale of jet growth. For the case $\mu_{\mathrm{d}}<\mu_{\mathrm{c}}$, the time scale of pinch-off was estimated as (Powers et al. 1998)

$t_{\mathrm{p}}=\frac{C R_{\mathrm{jet}} \mu_{\mathrm{c}}}{\sigma}$

where $C$ is a constant depending on the viscosity ratio and $R_{\text {jet }}$ is the unperturbed jet radius. In the present study, $\mu_{\mathrm{d}} / \mu_{\mathrm{c}} \sim 1$; therefore, Eq. (1) can still be used as an approximation.

For the case of low Reynolds number, the radius of liquid jet co-flowing inside another immiscible liquid in a cylindrical capillary of radius $R_{\mathrm{c}}$ can be found from (Utada et al. 2005)

$\frac{Q_{\mathrm{d}}}{Q_{\mathrm{c}}}=\frac{\mu_{\mathrm{c}}}{\mu_{\mathrm{d}}} \frac{\varepsilon^{2}}{(1-\varepsilon)^{2}}+2 \frac{\varepsilon}{1-\varepsilon}$

where $\varepsilon=\frac{R_{\mathrm{jet}}^{2}}{R_{\mathrm{c}}^{2}}$. Solving Eq. (2) for $\varepsilon$ gives

$\varepsilon=\frac{R_{\text {jet }}^{2}}{R_{\mathrm{c}}^{2}}=\frac{S_{\text {jet }}}{S}=\frac{1+\frac{Q_{\mathrm{d}}}{Q_{\mathrm{c}}}-\sqrt{1+\frac{Q_{\mathrm{d}}}{Q_{\mathrm{c}}} \frac{\mu_{\mathrm{c}}}{\mu_{\mathrm{d}}}}}{2+\frac{Q_{\mathrm{d}}}{Q_{\mathrm{c}}}-\frac{\mu_{\mathrm{c}}}{\mu_{\mathrm{d}}}}$

where $S_{\text {jet }}$ is the area of jet cross-section and $S$ is the area of capillary cross-section. The cross-section of the channel

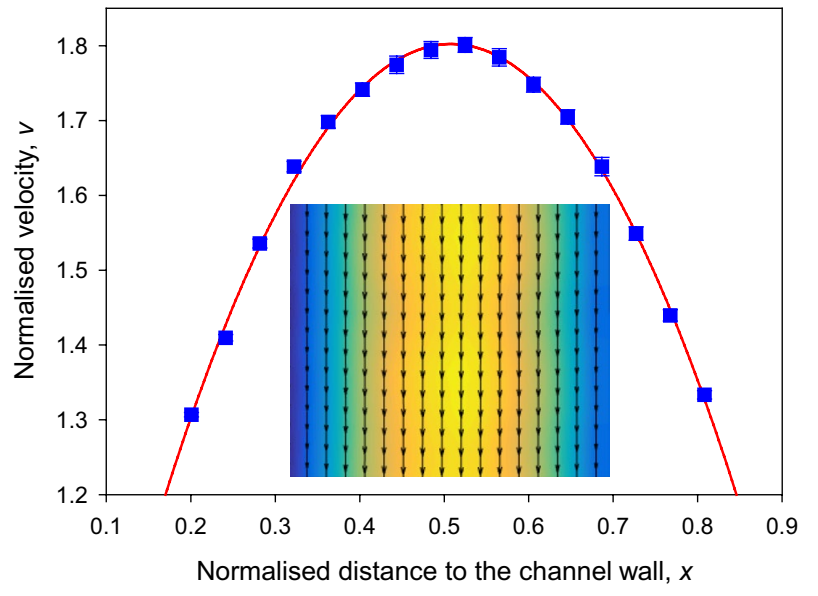

Fig. 4 Flow distribution in a stable jet of surfactant-free glycerolwater mixture at $Q_{\mathrm{c}}=10 \mu \mathrm{L} / \mathrm{min}\left(C a_{\mathrm{c}}=4.0 \times 10^{-4}\right), Q_{\mathrm{d}}=100 \mu \mathrm{L} / \mathrm{min}$ $\left(C a_{\mathrm{d}}=5.2 \times 10^{-3}\right)$ : the points are the average values of the velocity on each streamline, the curve is a parabolic fitting according to the equation $v=0.44+5.36 x-5.27 x^{2}$ with $R_{\text {sqr }}=0.998$. The jet is situated at $0.2 \leq x \leq 0.8$. The velocity is normalised by the superficial velocity in the output channel, $U_{\mathrm{o}}=30 \mathrm{~mm} / \mathrm{s}$

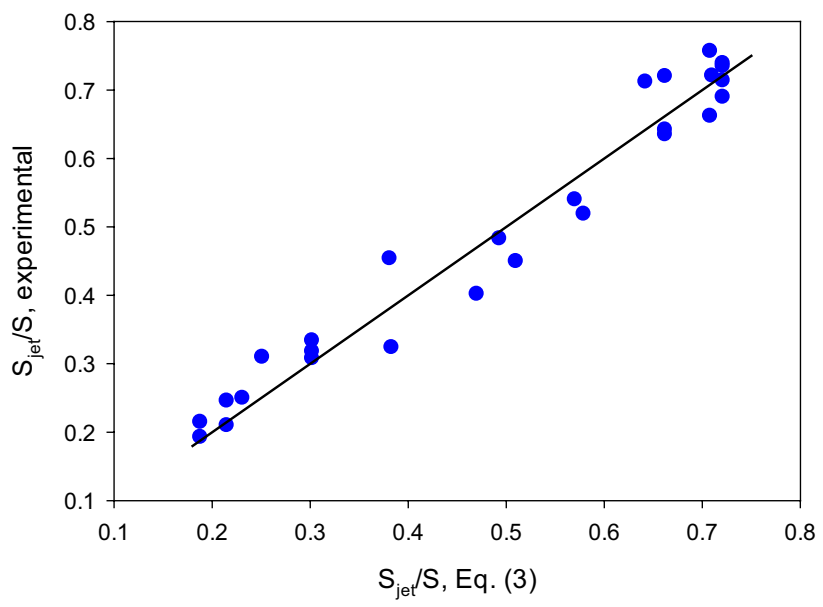

Fig. 5 Relation between the values of jet cross-section normalised by the channel cross-section, $S_{\text {jet }} / S$, calculated from Eq. (3) and found from the experimental jet images. The line corresponds to $S_{\text {jet }} / S$, Eq. (3) $=S_{\text {jet }} / S$, experimental

used in this study (Fig. 1) is not circular; nevertheless, the flow profile inside the stable jets is parabolic in agreement with the model used to derive Eq. (2) as shown in Fig. 4. A jet is considered to be stable if it does not break into drops inside the length of the output channel. It was assumed that the jets with a width smaller than the channel height are cylindrical, whereas those with a larger width fit the shape of the channel. Under this assumption, the series of measurements in the range of $0.5 \leq Q_{\mathrm{d}} / Q_{\mathrm{c}} \leq 10$ for jets formed by surfactant-free and surfactant-laden dispersed phases has shown that the values of $S_{\text {jet }} / S$ calculated from the corresponding 
images coincide with those found from Eq. 3 within an error of $20 \%$ (Fig. 5). The errors can be related not only to deviation of the channel geometry from the circular one, but also to some jet asymmetry in experiments and inaccuracy of the assumptions made above with respect to jet shape. Therefore, Eqs. (1) and (3) can be used for the estimation of pinch-off time scale.

Equation (3) also provides the opportunity to estimate the Reynolds numbers at the dripping to jetting transition using $Q_{\mathrm{d}} / S_{\text {jet }}$ and $2 Q_{\mathrm{c}} /\left(S-S_{\text {eft }}\right)$ as the characteristic velocity scale and $\sqrt{S_{\text {jet }} / \pi}$ and $\sqrt{\left(S-S_{\text {jet }}\right) / \pi}$ as characteristic length scale for the dispersed and continuous phases, respectively. The results of the calculations for the surfactant-free system and for the dispersed phase containing $\mathrm{C}_{12} \mathrm{TAB}$ surfactant are presented in Fig. 6 versus the capillary number of the continuous phase for convenience of comparison with Fig. 2. For the dispersed phase containing $50 \mathrm{mM}$ of TX-100 or di-BC $\mathrm{C}_{9} \mathrm{SG}$, the Reynolds numbers decrease further, with $R e<1$ for both the continuous and dispersed phases over the whole range of flow rates studied.

Comparison of Fig. 6 with Fig. 2a shows that the ascending branch in Fig. 2 a corresponds to the condition $R e_{\mathrm{d}}>R e_{\mathrm{c}}$, whereas the plateau and descending branch are characterised by $R e_{\mathrm{c}}>R e_{\mathrm{d}}$. The transition from plateau to descending branch occurs at $C a_{\mathrm{c}}$ where $R e_{\mathrm{d}}$ begins to decrease.

Despite the fact that $R e_{\mathrm{c}}>1$ for the larger range of flow rates in Fig. 6, the viscosity of the continuous phase is extremely important because it determines the velocity distribution inside the continuous phase and the shear stress imposed by the continuous phase at the water/oil interface. That is why the capillary number of the continuous phase rather than the Weber number is used in microfluidic

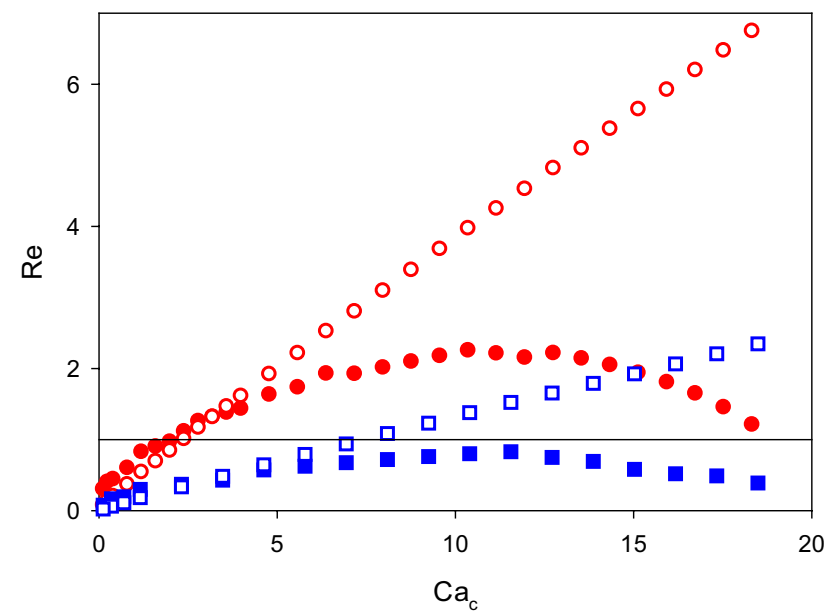

Fig. 6 Reynolds numbers of continuous (empty symbols) and dispersed (filled symbols) phases for the surfactant-free dispersed phase (circles) and dispersed phase with $50 \mathrm{mM}$ of $\mathrm{C} 12 \mathrm{TAB}$ (squares) at dripping $\rightarrow$ jetting transition studies (Utada et al. 2007; Christopher et al. 2008; Cubaud and Mason 2008; Castro-Hernández et al. 2009; Bertrandias et al. 2017; Deng et al. 2017; Wu et al. 2017). At the same time, for the dispersed phase both capillary (Cubaud and Mason 2008; Deng et al. 2017) and Weber (Utada et al. 2007; Castro-Hernández et al. 2009; Bertrandias et al. 2017; Wu et al. 2017) numbers are used. In the case considered here, the Reynolds number of the surfactant-free dispersed phase is of the order of unity, whereas for the surfactantladen dispersed phases, $R e_{\mathrm{d}}$ is always $<1$. Therefore, the capillary number was chosen as a characteristic dimensionless parameter for the dispersed phase.

The time scale of jet growth, $t_{\mathrm{g}}$, can be estimated as the time required for the jet length to become comparable to its radius (Utada et al. 2005):

$t_{\mathrm{g}}=\frac{\pi R_{\mathrm{jet}}^{3}}{Q_{\mathrm{d}}}$

Jetting regime occurs if $t_{\mathrm{g}}<t_{\mathrm{p}}$, i.e. $\frac{S_{\text {jet }}}{Q_{\mathrm{d}}}<\frac{C \mu_{\mathrm{c}}}{\sigma}$ or taking into account Eq. (3)

$\frac{S}{Q_{\mathrm{d}}} \frac{1+\frac{Q_{\mathrm{d}}}{Q_{\mathrm{c}}}-\sqrt{1+\frac{Q_{\mathrm{d}}}{Q_{\mathrm{c}}} \frac{\mu_{\mathrm{c}}}{\mu_{\mathrm{d}}}}}{2+\frac{Q_{\mathrm{d}}}{Q_{\mathrm{c}}}-\frac{\mu_{\mathrm{c}}}{\mu_{\mathrm{d}}}}<\frac{C \mu_{\mathrm{c}}}{\sigma}$.

For simplicity, let us consider the condition (5) for the case $\mu_{\mathrm{c}}=\mu_{\mathrm{d}}=\mu$ where the viscosity ratio is close to unity as considered here. The inequality (5) in this case simplifies to

$1-C a_{\mathrm{d}} C<\sqrt{\frac{Q_{\mathrm{c}}}{\left(Q_{\mathrm{c}}+Q_{\mathrm{d}}\right)}}$,

which is true for any $Q_{\mathrm{c}}$ in the case

$C a_{\mathrm{d}}>1 / C$

providing conditions for the plateau of Fig. 2a. Using $C=65$ obtained from numerical simulations for the case $\mu_{\mathrm{c}}=\mu_{\mathrm{d}}$ (Powers et al. 1998), one obtains the result that the jetting regime is expected for $C a_{\mathrm{d}}>0.015$, which is in reasonable agreement with Fig. 2a. If $C a_{\mathrm{d}}<1 / C$ then solving (5a) predicts jetting regime for

$C a_{\mathrm{c}}>\frac{\left(\frac{1}{C}-C a_{\mathrm{d}}\right)^{2}}{\frac{2}{C}-C a_{\mathrm{d}}}$.

Inequality (7) shows that the jetting regime is established when the capillary number exceeds a certain threshold value, increasing with a decrease of $C a_{\mathrm{d}}$, i.e. it describes the descending branch in Fig. 2a. For $C=65$, the threshold value of $C a_{\mathrm{c}}$ according to (7) increases from 0.003 at 
$C a_{\mathrm{d}}=0.006-0.007$ at $C a_{\mathrm{d}}=0.001$, as shown by the dashed line in Fig. 2a. With a decrease of $\mathrm{C}$, the slope of the line calculated from (7) increases and it moves to larger values of $C a_{\mathrm{c}}$. The best fitting of the descending branch is obtained for $C=25$ (solid line in Fig. 2a), however a decrease of $\mathrm{C}$ results in the shifting of $C a_{\text {dcrito }}$ to larger values. Taking into account all assumptions and simplifications present in the mathematical model, these results are in reasonably good agreement with the experiments.

Inequalities (6) and (7) have a clear physical meaning: if the velocity of dispersed phase is high enough then the jetting regime is driven by the flow of dispersed phase only, whereas at smaller velocities of dispersed phase, an additional contribution from the continuous phase results in a decrease of the jet radius and therefore an increasing velocity inside the dispersed phase. The smaller the velocity of dispersed phase becomes, the larger should be the contribution from the velocity of the continuous phase. Note, (5) does not provide any conditions for the ascending branch in Fig. 2a, which is now considered in more detail.

As shown in Fig. $3 \mathrm{a}$ and in the video S1, immediately before drop detachment the jet has a second neck inside the focusing channel. After drop detachment, the convex part of the jet retracts and the formation of the next drop starts. As the remaining neck is still situated inside the focusing part of the channel, initially the drop formation follows the root characteristic for the dripping regime (Fig. 7a, video $\mathrm{S} 1$ ). It is noticeable that the flow profile in the neck (the narrowest cross-section) is parabolic with a very good precision. The parabolic fitting is given by the equation $V=-44.8+287.4 x-292.1 x^{2}$ with (extrapolated) $V<0$ at the channel walls $(x=0$ and $x=1)$. Taking into account that at the high concentration of surfactant used in this study, dynamic effects of surfactant can be neglected and shear stress should be continuous at the interface, $\mu_{\mathrm{c}} \nabla V_{\mathrm{c}}=\mu_{\mathrm{d}} \nabla V_{\mathrm{d}}$. Therefore, the negative value of velocity at the wall obtained from the fitting of flow in the dispersed phase is in contradiction to the condition $\mu_{\mathrm{c}}<\mu_{\mathrm{d}}$, because according to the tangential stress balance at the water/oil interface, the velocity in the continuous phase should change faster than in the dispersed phase (Yiantsios and Higgins 1988; Cubaud and Mason 2009). To obtain a zero flow velocity of the continuous phase on the wall, extrapolation of the velocity profile in the dispersed phase to the wall should give small positive value (sf. Fig. 4). Thus, a negative value demonstrates that, contrary to the case of a stable jet, the flow of continuous phase around the forming drop is not fully developed, i.e. there is no hydrodynamic equilibrium and the velocity gradient of the continuous phase should be smaller than that of dispersed phase.

Downstream of the neck, where the new drop is formed, the velocity of the continuous phase decreases due to an increase of available channel cross-section, but so does the velocity of the dispersed phase resulting in the drag force from continuous phase moving both drop and the neck position downstream. For example, the average velocity of the dispersed phase in the cross-section of maximum drop width in Fig. $7 \mathrm{a}$ is around $5.7 \mathrm{~mm} / \mathrm{s}$, whereas the average velocity of the continuous phase calculated from geometrical considerations is around $7.5 \mathrm{~mm} / \mathrm{s}$. If the drag force is relatively small, then the neck can move out of the narrow focusing part of geometry into the wider main output channel before drop detachment. As the channel cross-section increases, the neck diameter stabilises and a jet is formed.

To confirm this mechanism, the neck kinetics for dripping and jetting regimes were compared at flow rates of the dispersed and continuous phases where both regimes coexist (Fig. 8). Initially, both kinetics are rather similar. The neck thinning accelerates for the drop formed in the dripping regime at $\mathrm{t} \sim 20 \mathrm{~ms}$ after transition to the pinch-off stage of drop formation. The beginning of the pinch-off stage corresponds to the critical neck diameter below which the capillary pressure in the neck becomes larger than that in the drop resulting in system instability and fast drop detachment. In Fig. 8, the pinch-off stage can be identified by the sharp acceleration of the neck kinetics.

For the dripping regime, the critical value of the neck diameter in Fig. 6 was reached while the neck was still in the focusing part of the channel. Conversely, for the jetting regime, the neck left the focusing part of the channel just before the critical diameter was reached. Afterwards, the neck thinning slowed down to adapt to the increasing channel width and a drop was formed much later in the jetting regime.

Thus, it can be concluded that the jetting regime occurring at small capillary numbers of both the dispersed and continuous phases corresponding to the ascending branch in Fig. 2a is geometry mediated. It should appear only in flowfocusing devices with geometrical focusing, when the forming neck elongates beyond the focusing part. This conclusion is in line with the literature data: transition from the dripping to tubing regime for an increase of $C a_{\mathrm{d}}$ in the range of low $C a_{\mathrm{c}}$ was observed in devices with hydrodynamic focusing (Cubaud and Mason 2008; Fu et al. 2012; Wu et al. 2017), whereas transition to drop formation in jetting regime was found using a combination of hydrodynamic and geometrical focusing (Shahriari et al. 2016). If the flow rate of the continuous phase increases, the drag force increases. Therefore, drops will detach faster and at a certain value of the continuous phase flow rate, detachment happens when the neck is still inside the focusing part, resulting in transition from jetting to dripping.

It should be stressed that the size of the drops formed in the jetting regime at low $C a_{\mathrm{c}}$ is much larger than the jet diameter. Therefore, it can be assumed that drop detachment is caused not by jet instability, but rather by the drag 
Fig. 7 Velocity distribution in the dispersed phase during the drop formation in jetting regime at low $C a_{\mathrm{c}}$ for $50 \mathrm{mM}$ Triton-X100 in dispersed phase, $Q_{\mathrm{c}}=8 \mu \mathrm{L} / \mathrm{min}$ $\left(C a_{\mathrm{c}}=2.6 \times 10^{-3}\right), Q_{\mathrm{d}}=9 \mu \mathrm{L} /$ $\min \left(C a_{\mathrm{d}}=3.8 \times 10^{-3}\right): \mathbf{a} 6 \mathrm{~ms}$ and $\mathbf{b} 90 \mathrm{~ms}$ after the previous pinch-off. Position A is a node, whereas position $B$ is an antinode. The velocities on the colour scale are given in $\mathrm{mm} / \mathrm{s}$

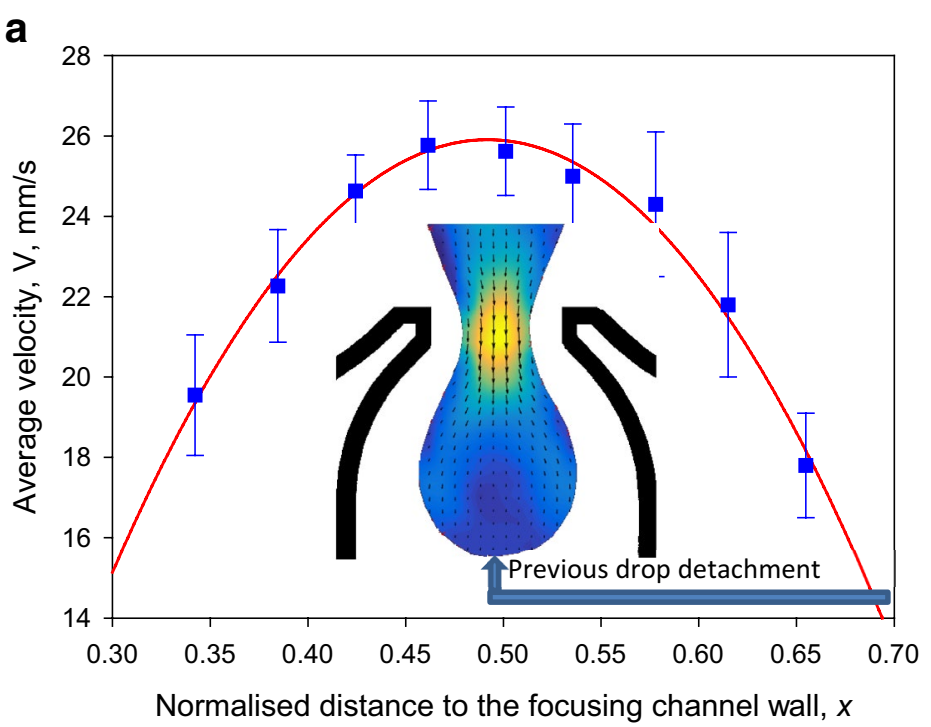

b

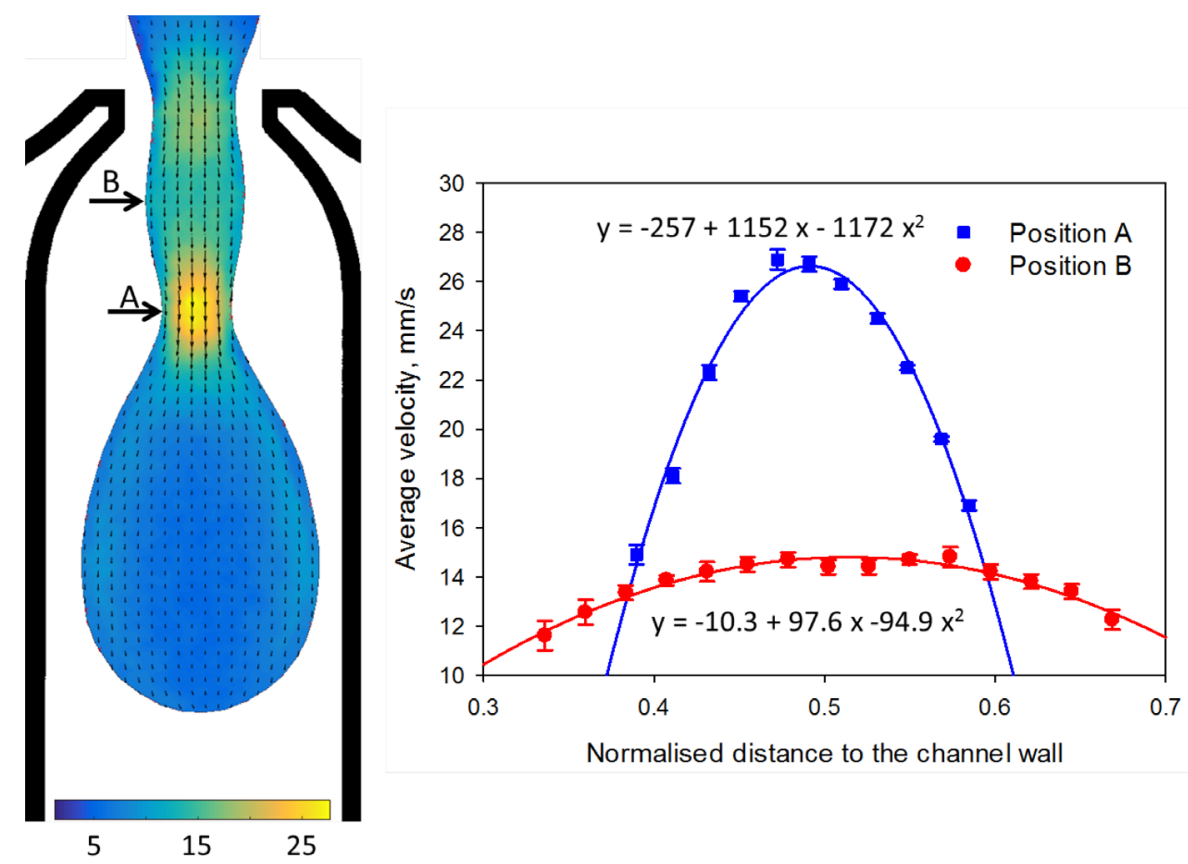

force from the continuous phase: when a drop grows large enough, the drag force exceeds the surface tension force and the drop detaches.

To test this hypothesis, for the drop presented in Fig. 7, the surface tension force at the neck and the drag force at the beginning of pinch-off stage are compared. From the neck kinetics corresponding to Fig. 7 (not shown), the critical neck diameter at the transition to pinch-off can be estimated as $d_{n} \sim 60 \mu \mathrm{m}$, similar to that in Fig. 8. Therefore, the surface tension force opposing the drop detachment is $F_{\sigma} \sim 6.8 \times 10^{-7} \mathrm{~N}$. The drag force acting on the drop can be roughly estimated using the modified Stokes equation proposed in Umbanhowar et al. (2000): $F_{\mathrm{d}}=3 \pi \mu_{\mathrm{c}}\left(d_{\mathrm{d}}-d_{n}\right)$ $\left(v_{\mathrm{c}}-v_{\mathrm{d}}\right), d_{\mathrm{d}}=290 \mu \mathrm{m}$ is the drop diameter at the beginning of the pinch-off stage, $v_{\mathrm{c}}$ is the velocity of the continuous phase at the drop surface and $v_{\mathrm{d}}$ is the longitudinal component of the drop velocity. The velocities can be estimated from the known flow rates and areas occupied by the dispersed and continuous phases. Assuming that the velocity of the continuous phase changes linearly between the wall and the surface of the drop, the velocity can be estimated as $v_{\mathrm{c}}=48.4 \mathrm{~mm} / \mathrm{s}$ and $v_{\mathrm{d}}=2.7 \mathrm{~mm} / \mathrm{s}$; the last value is in good agreement with Fig. 7b, and the drag force can be estimated as $F_{\mathrm{d}} \sim 4.6 \times 10^{-7} \mathrm{~N}$. Considering the very rough 


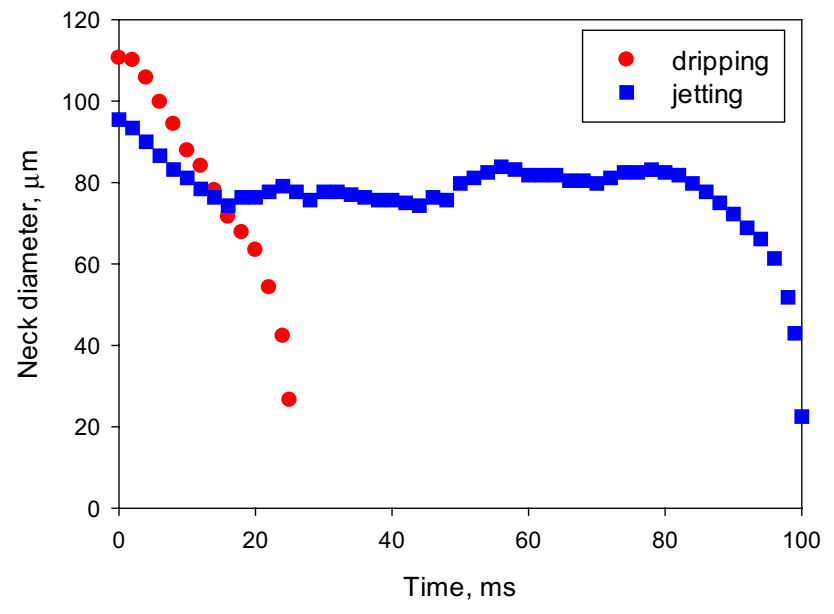

Fig. 8 Comparative neck kinetics at dripping and jetting regime. Dispersed phase is $50 \mathrm{mM}$ solution of Triton-X100, $Q_{\mathrm{c}}=6 \mu \mathrm{L} / \mathrm{min}$ $\left(C a_{\mathrm{c}}=1.9 \times 10^{-3}\right), Q_{\mathrm{d}}=9 \mu \mathrm{L} / \mathrm{min}\left(C a_{\mathrm{d}}=3.8 \times 10^{-3}\right)$

approximations used to calculate the drag force, this value is in good agreement with the surface tension force. Therefore, the assumption about the drag being the main driving force for drop detachment is quite plausible.

In this case, the drop formation around the ascending branch occurs nearly always at the same position and the drop size distribution is rather narrow. Therefore, the jetting regime at small $C a_{\mathrm{c}}$ can be used for the production of large but still quite monodisperse drops. The similarity of the drop formation mechanisms in the jetting regime dominated by the flow of dispersed phase and dripping regime was found in numerical simulations for co-flow (Chen et al. 2013). In particular, it was shown in Chen et al. (2013) that drop detachment in the dripping regime and in the jetting regime driven by the flow of the dispersed phase occurs due to high excessive capillary pressure in the neck, whereas drop detachment in the jetting regime mediated by the flow of the continuous phase is due to a lateral velocity gradient inside the jet.

As already mentioned, the transition jetting $\rightarrow$ dripping $\rightarrow$ jetting was predicted theoretically for co-flow in confined geometry (Guillot et al. 2007). At low flow rates of the continuous phase, the transition from jetting to dripping was ascribed to a reduction of the jet confinement enabling faster development of an instability. This mechanism is applicable for the case considered here: the decrease of confinement accelerates the instability development at small $C a_{\mathrm{c}}$ and, therefore, when $C a_{\mathrm{c}}$ increases the drop becomes unstable while the neck is still inside the focusing part of the channel, i.e. an increase in $C a_{\mathrm{c}}$ results in the transition to the dripping regime.

It should be stressed that over all the timescales of jet evolution at small $C a_{\mathrm{c}}$ the velocity profiles inside it remain parabolic (Fig. 7b), with (extrapolated) large negative values

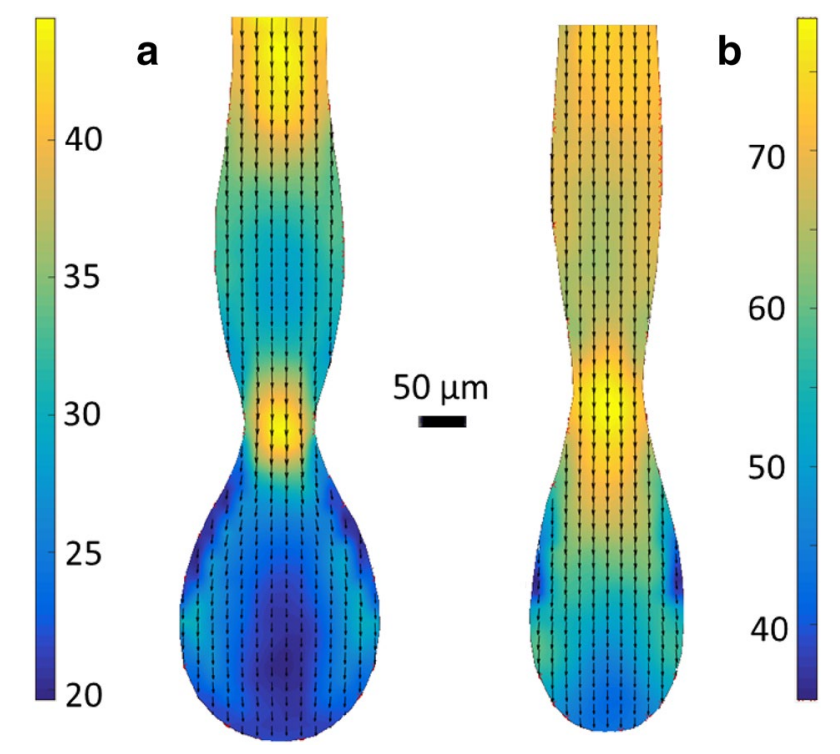

Fig. 9 Velocity distribution during the drop formation in jetting regime at intermediate and high $C a_{\mathrm{c}}$ for $50 \mathrm{mM}$ Triton-X100 in dispersed phase: a $Q_{\mathrm{c}}=30 \mu \mathrm{L} / \mathrm{min}\left(C a_{\mathrm{c}}=9.6 \times 10^{-3}\right), Q_{\mathrm{d}}=20 \mu \mathrm{L} / \mathrm{min}$ $\left(C a_{\mathrm{d}}=8.3 \times 10^{-3}\right), \mathbf{b} Q_{\mathrm{c}}=60 \mu \mathrm{L} / \mathrm{min}\left(C a_{\mathrm{c}}=19.2 \times 10^{-3}\right), Q_{\mathrm{d}}=40 \mu \mathrm{L} /$ $\min \left(C a_{\mathrm{d}}=16.6 \times 10^{-3}\right)$. The velocities of the colour scale are given in $\mathrm{mm} / \mathrm{s}$

at the wall, i.e. the flow of the dispersed phase dominates the flow of the continuous phase (see also Fig. 6). However, the velocity profiles in the jets forming around the plateau and descending branch of Fig. 2a can be even reversed, with velocity decreasing towards the jet axis in the convex part of the jet, antinode, (Fig. 9), identifying the growing importance of the continuous phase contribution inside the horizontal branch. This implies that the contribution from the continuous phase becomes noticeable inside the plateau, although it becomes dominant only on the descending branch (Figs. 2b, 6).

Drop formation in the jetting regime mediated by the continuous phase is due to the Rayleigh-Plateau instability. Often in this case, the jet does not break at each node (Fig. 10a, video S5), resulting in a multimodal drop size distribution. Moreover, drops formed in the dripping regime under the condition $C a_{\mathrm{c}} \gg C a_{\mathrm{d}}$ are accompanied by multiple satellite drops of various sizes (Fig. 10b), some of them of comparable size to the main drop. Therefore, these conditions are unfavourable for the production of monodisperse drops. Satellite drops have been observed over the whole range of flow rates in both dripping and jetting regimes. However, in most cases they are much smaller than the main drop (sf. Figs. 3, 10b).

If drop formation results from the Rayleigh-Plateau instability, it was found that the drop radius is proportional to the jet radius and $\sim Q_{\mathrm{d}} / Q_{\mathrm{c}}^{0.5}$ for both co-flow and hydrodynamic flow focusing (Utada et al. 2007; Cubaud and Mason 2008). 

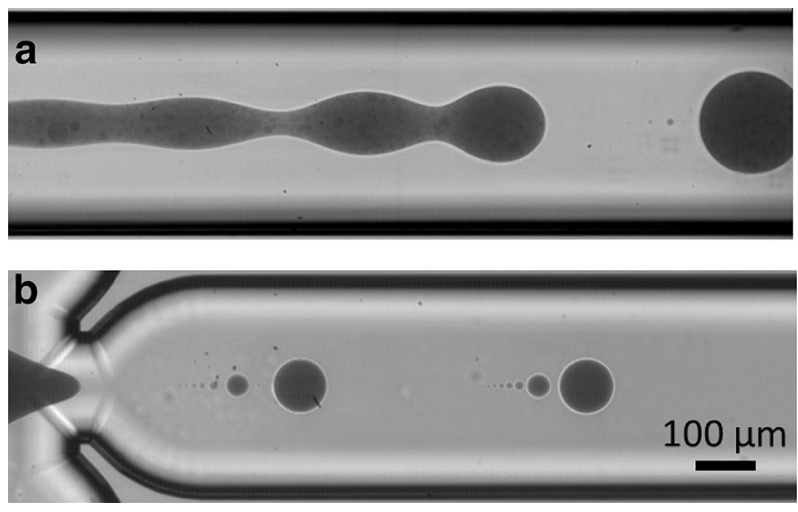

Fig. 10 Drop formation in jetting (a) and dripping (b) regime mediated by continuous phase: a dispersed phase $50 \mathrm{mM}$ solution of di-BC ${ }_{9} \mathrm{SG}, Q_{\mathrm{c}}=40 \mu \mathrm{L} / \mathrm{min}\left(C a_{\mathrm{c}}=33.0 \times 10^{-3}\right), Q_{\mathrm{d}}=10 \mu \mathrm{L} /$ $\min \left(C a_{\mathrm{d}}=10.8 \times 10^{-3}\right), \mathbf{b}$ dispersed phase $50 \mathrm{mM}$ solution of Triton-X100, $Q_{\mathrm{c}}=60 \mu \mathrm{L} / \mathrm{min} \quad\left(C a_{\mathrm{c}}=19.2 \times 10^{-3}\right), Q_{\mathrm{d}}=2 \mu \mathrm{L} / \mathrm{min}$ $\left(C a_{\mathrm{d}}=0.8 \times 10^{-3}\right)$

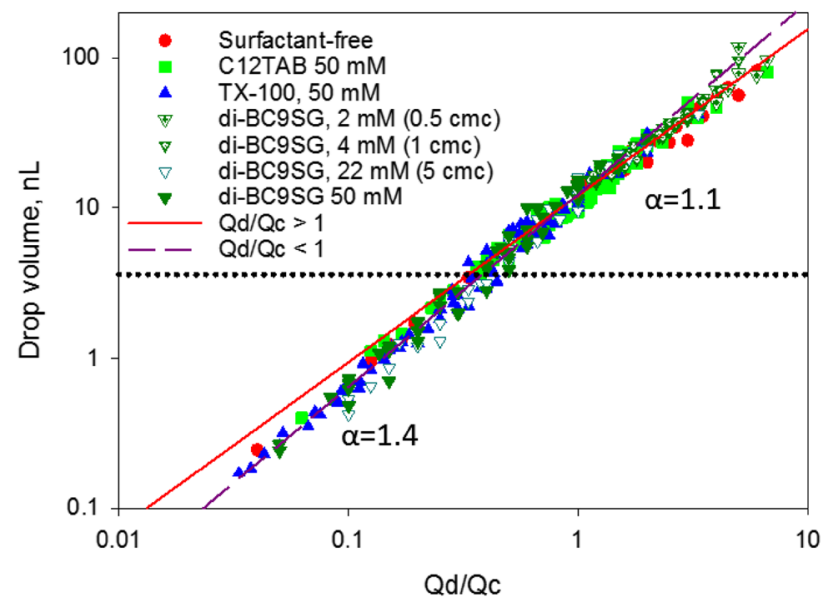

Fig. 11 Drop size versus flow rate ratio for drop formation in jetting regime. The solid and dashed lines correspond to the linear regression fitting. The dotted horizontal line corresponds to the drop size equal to the channel height

For the case when drop formation in the jetting regime is mediated by the dispersed phase (co-flow) $R_{\text {drop }} \sim Q_{\mathrm{d}} / Q_{\mathrm{c}}^{0.46}$ (Utada et al. 2007). The device used by Utada et al. (2007) has a circular cross-section and the device used by Cubaud and Mason (2008) has a square cross-section, whereas the channel used in this study has a difference between the width and height. Therefore, in this work the changes in the observed drop length depend on the drop shape. In particular, the drops formed at low $C a_{\mathrm{c}}$ have a pancake-shape (1-D confinement by the channel height, only drop length and width can change) or plug (2-D confinement by the channel height and width, only drop length can change). To make a consistent comparison of drop size, the drop volume, which was found from the known flow rate of the dispersed phase and the frequency of drop formation, is presented in Fig. 11 as a function of the flow rate ratio. Figure 11 presents 307 data points including those for concentrations of the surfactant di-BC ${ }_{9} \mathrm{SG}$ smaller than $50 \mathrm{mM}$, where the surfactant dynamic should be of importance. It is seen from Fig. 11 that independently of surfactant type and concentration all data fall onto the same master curve, a power law with an exponent $\alpha \sim 1.4$ for $Q_{\mathrm{d}} / Q_{\mathrm{c}}<1$ and $\alpha \sim 1.1$ for $Q_{\mathrm{d}} / Q_{\mathrm{c}}>1$. This result is in good agreement with (Utada et al. 2007; Cubaud and Mason 2008), where the scaling exponent for the volume should be in the range 1.38-1.5. A smaller exponent value at $Q_{\mathrm{d}} / Q_{\mathrm{c}}>1$ is most probably due to the effect of geometrical confinement on drop formation, which was absent in Utada et al. (2007) and Cubaud and Mason (2008). The transition to the smaller exponent occurs when the drop size becomes noticeably larger than the channel height, see the dotted line in Fig. 11.

\section{Conclusions}

A study of drop formation in a microfluidic flow-focusing device was carried out for a surfactant-free and three surfactant-laden dispersed phases under the condition of similar viscosities of continuous and dispersed phases. It has been shown that the transition from dripping to jetting for all studied dispersed phases occurs along similar transition curves in coordinates of capillary numbers of continuous and dispersed phase. The jetting regime was only observed when the capillary number of dispersed phase, $C a_{\mathrm{d}}$, exceeded a critical value $C a_{\text {dcrito }}$. This critical value can be derived from the comparison of the characteristic time scale of drop pinch-off with the time scale of jet growth.

If $C a_{\mathrm{d}}<C a_{\mathrm{dcrit} 0}$ an increase in the capillary number of the continuous phase results in a jetting $\rightarrow$ dripping $\rightarrow$ jetting transition. This is the first observation of such transition in a flow-focusing device.

The jetting regime at large $C a_{\mathrm{c}}$ is mediated by the continuous phase flow rate with $C a_{\text {dcrit }}$ decreasing with an increase of $C a_{\mathrm{c}}$ from $C a_{\mathrm{dcrit} 0}$ to at least $C a_{\text {dcrito }} / 10$. This descending branch of the transition line can also be derived from the comparison of the characteristic time scale of drop pinchoff with the time scale of jet growth. In the jetting regime at large $C a_{\mathrm{c}}$, drops are formed due to the Rayleigh-Plateau instability often with multimodal size distribution. The flow field inside the jet is parabolic in nodes and reversed parabolic (with minimum flow rate at the axis) in antinodes.

The jetting regime at small $C a_{\mathrm{c}}$ is mediated by the dispersed phase flow rate with $C a_{\text {dcrit }}$ increasing with an increase of $C a_{\mathrm{c}}$ from $\sim C a_{\text {dcrito }} / 10$ to $C a_{\text {dcrito. }}$. This ascending branch of the transition line cannot be derived from the comparison of the characteristic time scale of drop pinch-off with the time scale of jet growth. It is suggested that it is 
geometry mediated and the drop detachment mechanism is similar to that in the dripping regime resulting in a narrow size distribution. The flow field inside the jet is parabolic in both nodes and antinodes.

The volume of the drops produced in the jetting regime at a similar flow rate ratio is independent of interfacial tension and any dynamic effects due to surfactant redistribution. It depends only on flow rate ratio with the power law exponent $\alpha \sim 1.4$ being close to the theoretical prediction for the jetting regime at $Q_{\mathrm{d}} / Q_{\mathrm{c}}<1$. The slightly smaller value of the exponent $\alpha \sim 1.1$ at $Q_{\mathrm{d}} / Q_{\mathrm{c}}>1$ is most probably related to the effect of confinement on drop formation in the microchannel.

Acknowledgements This work was funded by the EPSRC Programme Grant "MEMPHIS - Multiscale Examination of Multiphase Physics in Flows" (EP/K003976/1), the Royal Society Research Grant 2017 R1 (RG160698), JSPS [KAKENHI, Fund for the Promotion of Joint International Research (Fostering Joint International Research) No. 15KK0221, Grant-in-Aid for Scientific Research (B), No. 19H02504], and by Procter \& Gamble. K. Steponavicius acknowledges the University of Birmingham for financial support of his summer research project.

\section{Compliance with ethical standards}

Conflict of interest The authors declare that they have no conflict of interest.

Open Access This article is distributed under the terms of the Creative Commons Attribution 4.0 International License (http://creativeco mmons.org/licenses/by/4.0/), which permits unrestricted use, distribution, and reproduction in any medium, provided you give appropriate credit to the original author(s) and the source, provide a link to the Creative Commons license, and indicate if changes were made.

\section{References}

Ambravaneswaran B, Subramani HJ, Phillips SD, Basaran OA (2004) Dripping-jetting transitions in a dripping faucet. Phys Rev Lett 93(3):034501

Anna SL (2016) Droplets and bubbles in microfluidic devices. Ann Rev Fluid Mech 48(1):285-309

Anna SL, Mayer HC (2006) Microscale tipstreaming in a microfluidic flow focusing device. Phys Fluids 18(12):121512

Anna SL, Bontoux N, Stone HA (2003) Formation of dispersions using "flow focusing" in microchannels. Appl Phys Lett 82(3):364-366

Bertrandias A, Duval H, Casalinho J, Giorgi ML (2017) Dripping to jetting transition for cross-flowing liquids. Phys Fluids 29(4):044102

Buzzaccaro S, Secchi E, Piazza R (2013) Ghost particle velocimetry: accurate 3D flow visualization using standard lab equipment. Phys Rev Lett 111(4):048101

Capretto L, Mazzitelli S, Nastruzzi C (2012) Design, production and optimization of solid lipid microparticles (SLM) by a coaxial microfluidic device. J Control Release 160(3):409-417

Carrier O, Ergin FG, Li H-Z, Watz BB, Funfschilling D (2015) Timeresolved mixing and flow-field measurements during droplet formation in a flow-focusing junction. J Micromech Microeng 25(8):084014
Castro-Hernández E, Gundabala V, Fernández-Nieves A, Gordillo JM (2009) Scaling the drop size in coflow experiments. New J Phys 11(7):075021

Chen Y, Wu L, Zhang C (2013) Emulsion droplet formation in coflowing liquid streams. Phys Rev E Stat Nonlinear Soft Matter Phys 87(1):013002

Chen X, Glawdel T, Cui N, Ren CL (2015) Model of droplet generation in flow focusing generators operating in the squeezing regime. Microfluid Nanofluid 18(5-6):1341-1353

Christopher GF, Noharuddin NN, Taylor JA, Anna SL (2008) Experimental observations of the squeezing-to-dripping transition in T-shaped microfluidic junctions. Phys Rev E Stat Nonlinear Soft Matter Phys 78(3 Pt 2):036317

Clanet C, Lasheras JC (1999) Transition from dripping to jetting. J Fluid Mech 383:307-323

Cordero ML, Gallaire F, Baroud CN (2011) Quantitative analysis of the dripping and jetting regimes in co-flowing capillary jets. Phys Fluids 23(9):094111

Cramer C, Fischer P, Windhab EJ (2004) Drop formation in a co-flowing ambient fluid. Chem Eng Sci 59(15):3045-3058

Cubaud T, Mason TG (2008) Capillary threads and viscous droplets in square microchannels. Phys Fluids 20(5):053302

Cubaud T, Mason TG (2009) High-viscosity fluid threads in weakly diffusive microfluidic systems. New J Phys 11(7):075029

de Saint Vincent MR, Delville J-P (2016) Fragmentation mechanisms of confined co-flowing capillary threads revealed by active flow focusing. Phys Rev Fluids 1(4):043901

DeMello AJ (2006) Control and detection of chemical reactions in microfluidic systems. Nature 442(7101):394-402

Deng C, Wang H, Huang W, Cheng S (2017) Numerical and experimental study of oil-in-water $(\mathrm{O} / \mathrm{W})$ droplet formation in a coflowing capillary device. Colloids Surf A 533:1-8

Fu T, Wu Y, Ma Y, Li HZ (2012) Droplet formation and breakup dynamics in microfluidic flow-focusing devices: from dripping to jetting. Chem Eng Sci 84:207-217

Garstecki P, Stone HA, Whitesides GM (2005) Mechanism for flowrate controlled breakup in confined geometries: a route to monodisperse emulsions. Phys Rev Lett 94(16):164501

Garstecki P, Fuerstman MJ, Stone HA, Whitesides GM (2006) Formation of droplets and bubbles in a microfluidic T-junction-scaling and mechanism of break-up. Lab Chip 6(3):437-446

Glawdel T, Elbuken C, Ren CL (2012) Droplet formation in microfluidic T-junction generators operating in the transitional regime. I. Experimental observations. Phys Rev E Stat Nonlinear Soft Matter Phys 85(1 Pt 2):016322

Guillot P, Colin A, Utada AS, Ajdari A (2007) Stability of a jet in confined pressure-driven biphasic flows at low reynolds numbers. Phys Rev Lett 99(10): 104502

Herrada MA, Ganan-Calvo AM, Guillot P (2008) Spatiotemporal instability of a confined capillary jet. Phys Rev E Stat Nonlinear Soft Matter Phys 78(4 Pt 2):046312

Holtze C, Weisse SA, Vranceanu M (2017) Commercial value and challenges of drop-based microfluidic screening platforms-an opinion. Micromachines 8(6):193

Kovalchuk NM, Chowdhury J, Schofield Z, Vigolo D, Simmons MJH (2018a) Study of drop coalescence and mixing in microchannel using Ghost Particle Velocimetry. Chem Eng Res Des 132:881-889

Kovalchuk NM, Roumpea E, Nowak E, Chinaud M, Angeli P, Simmons MJH (2018b) Effect of surfactant on emulsification in microchannels. Chem Eng Sci 176:139-152

Lee W, Walker LM, Anna SL (2009) Role of geometry and fluid properties in droplet and thread formation processes in planar flow focusing. Phys Fluids 21(3):032103

Marre S, Jensen KF (2010) Synthesis of micro and nanostructures in microfluidic systems. Chem Soc Rev 39(3):1183-1202 
Mastiani M, Seo S, Jimenez SM, Petrozzi N, Kim MM (2017) Flow regime mapping of aqueous two-phase system droplets in flowfocusing geometries. Colloids Surf A 531:111-120

Mitropoulos AN, Perotto G, Kim S, Marelli B, Kaplan DL, Omenetto FG (2014) Synthesis of silk fibroin micro- and submicron spheres using a co-flow capillary device. Adv Mater 26(7):1105-1110

Pirbodaghi T, Vigolo D, Akbari S, deMello A (2015) Investigating the fluid dynamics of rapid processes within microfluidic devices using bright-field microscopy. Lab Chip 15(9):2140-2144

Powers TR, Zhang D, Goldstein RE, Stone HA (1998) Propagation of a topological transition: the Rayleigh instability. Phys Fluids 10(5):1052-1057

Rakszewska A, Tel J, Chokkalingam V, Huck WTS (2014) One drop at a time: toward droplet microfluidics as a versatile tool for singlecell analysis. NPG Asia Mater 6(10):e133

Riccomi M, Alberini F, Brunazzi E, Vigolo D (2018) Ghost Particle Velocimetry as an alternative to $\mu$ PIV for micro/milli-fluidic devices. Chem Eng Res Des 133:183-194

Sagisaka M, Koike D, Mashimo Y, Yoda S, Takebayashi Y, Furuya T, Yoshizawa A, Sakai H, Abe M, Otake K (2008) Water/supercritical $\mathrm{CO}_{2}$ microemulsions with mixed surfactant systems. Langmuir 24(18):10116-10122

Sagisaka M, Narumi T, Niwase M, Narita S, Ohata A, James C, Yoshizawa A, Taffin de Givenchy E, Guittard F, Alexander S, Eastoe J (2014) Hyperbranched hydrocarbon surfactants give fluorocarbonlike low surface energies. Langmuir 30(21):6057-6063

Schneider CA, Rasband WS, Eliceiri KW (2012) NIH Image to ImageJ: 25 years of image analysis. Nat Methods 9(7):671-675

Shahriari A, Kim MM, Zamani S, Phillip N, Nasouri B, Hidrovo CH (2016) Flow regime mapping of high inertial gas-liquid droplet microflows in flow-focusing geometries. Microfluid Nanofluid 20(1):20

Thielicke W, Stamhuis EJ (2014) PIVlab-towards user-friendly, affordable and accurate digital particle image velocimetry in MATLAB. J Open Res Softw 2:p.e30
Umbanhowar PB, Prasad V, Weitz DA (2000) Monodisperse emulsion generation via drop break off in a coflowing stream. Langmuir 16:347-351

Utada AS, Lorenceau E, Link DR, Kaplan DL, Stone HA, Weitz DA (2005) Monodisperse double emulsions generated from a microcapillary device. Science 308:537-541

Utada AS, Fernandez-Nieves A, Stone HA, Weitz DA (2007) Dripping to jetting transitions in coflowing liquid streams. Phys Rev Lett 99(9):094502

Utada AS, Fernandez-Nieves A, Gordillo JM, Weitz DA (2008) Absolute instability of a liquid jet in a coflowing stream. Phys Rev Lett 100(1):014502

van Steijn V, Kleijn CR, Kreutzer MT (2010) Predictive model for the size of bubbles and droplets created in microfluidic T-junctions. Lab Chip 10(19):2513-2518

Vladisavljević G, Al Nuumani R, Nabavi S (2017) Microfluidic production of multiple emulsions. Micromachines 8(12):75

Ward T, Faivre M, Stone HA (2010) Drop production and tip-streaming phenomenon in a microfluidic flow-focusing device via an interfacial chemical reaction. Langmuir 26(12):9233-9239

Whitesides GM (2006) The origins and the future of microfluidics. Nature 442(7101):368-373

Wu Z, Cao Z, Sundén B (2017) Liquid-liquid flow patterns and slug hydrodynamics in square microchannels of cross-shaped junctions. Chem Eng Sci 174:56-66

Yiantsios SG, Higgins BG (1988) Linear stability of plane Poiseuille flow of two superposed fluids. Phys Fluids 31(11):3225-3238

Publisher's Note Springer Nature remains neutral with regard to jurisdictional claims in published maps and institutional affiliations. 